E. Russell Love, Department of Mathematics, University of Melbourne, Parkville, Victoria 3052, Australia

\title{
INTEGRATION OF FOURIER-LEGENDRE SERIES
}

\begin{abstract}
It is well known than an ordinary (trigonometric) Fourier series "can" be integrated term by term; that is, whether the Fourier series of an integrable function $f$ is convergent or not, the series obtained by term by term integration of it is convergent to the corresponding integral of $f$. There is also a more general theorem in which $f$ and its Fourier series are first multiplied by a function of bounded variation before integrating. This paper aims to obtain similar theorems for the Fourier-Legendre series discussed by Love and Hunter in Proc. London Math. Soc (3) 64 (1992) 579-601 and by Love in that journal (3) 69 (1994) 629-672. Most of the paper is occupied by lemmas which lead to establishing the dominated convergence of the partial sums of the Fourier-Legendre series under certain conditions. For several reasons one of these conditions is that the interval of integration must be a closed subinterval of $(-1,1)$, the interval on which the Legendre functions $P_{\nu}^{\mu}(x)$ are defined; further, I have only had success with integration over subintervals of $\left[-\frac{1}{2} \sqrt{3}, \frac{1}{2} \sqrt{3}\right]$
\end{abstract}

\section{Introduction}

There is a well-known theorem [3] (p. 419) that a (trigonometric) Fourier series "can" be integrated term-by-term. This means that if a function $f$ has

Key Words: Fourier-Legendre series, bounded variation, the Dini condition

Mathematical Reviews subject classification: 26A33

Received by the editors July 26,1995

* This paper was written for the 19th Summer Symposium in Real Analysis, held at Erice, Sicily in June 1995, and my thanks are due to the organizers for providing a spur towards its creation. 
the following Fourier series; that is, if

$$
f(x) \sim \frac{1}{2} \alpha_{o}+\sum_{n=1}^{\infty}\left(\alpha_{n} \cos n x+\beta_{n} \sin n x\right),
$$

whether the series is convergent or not, then

$$
\int_{a}^{b} f(x) d x=\frac{1}{2} \alpha_{o} \int_{a}^{b} d x+\sum_{n=1}^{\infty}\left(\alpha_{n} \int_{a}^{b} \cos n x d x+\beta_{n} \int_{a}^{b} \sin n x d x\right)
$$

More generally [3] (p. 421), any factor $g(x)$ of bounded variation on $[a, b]$ can be inserted into the integrands, giving again a correct equation

$$
\begin{gathered}
\int_{a}^{b} f(x) g(x) d x= \\
\frac{1}{2} \alpha_{o} \int_{a}^{b} g(x) d x+\sum_{n=1}^{\infty}\left(\alpha_{n} \int_{a}^{b} g(x) \cos n x d x+\beta_{n} \int_{a}^{b} g(x) \sin n x d x\right) .
\end{gathered}
$$

This paper aims to find similar theorems for the Fourier-Legendre series studied by Hunter and me [2], in which

$$
\begin{gathered}
f(x) \sim \sum_{n=-\infty}^{\infty} a_{n} P_{\nu+n}^{\mu}(x) \\
\text { where } a_{n}=(-1)^{n} \frac{\nu+n+\frac{1}{2}}{2 \cos \nu \pi} \int_{-1}^{1} f(t) P_{\nu+n}^{-\mu}(-t) d t .
\end{gathered}
$$

Such a theorem might be as follows.

Proposition 1. If $0<c<1, f \in L(-c, c)$, $f$ vanishes outside $(-c, c), g$ has bounded variation on $[-c, c]$ and $f$ has the Fourier-Legendre series (1), is it true that

$$
\int_{-c}^{c} f(x) g(x) d x=\sum_{-\infty}^{\infty} a_{n} \int_{-c}^{c} P_{\nu+n}^{\mu}(x) g(x) d x ?
$$

Partial Proof. By the definition of $a_{r}$ in (1),

$$
\begin{gathered}
\sum_{r=-n}^{n-1} a_{r} \int_{-c}^{c} P_{\nu+r}^{\mu}(x) g(x) d x \\
\sum_{r=-n}^{n-1}(-1)^{r} \frac{\nu+r+\frac{1}{2}}{2 \cos \nu \pi} \int_{-c}^{c} f(t) P_{\nu+r}^{-\mu}(-t) d t \int_{-c}^{c} P_{\nu+r}^{\mu}(x) g(x) d x
\end{gathered}
$$




$$
\begin{gathered}
\int_{-c}^{c} f(t)\left(\sum_{r=-n}^{n-1} P_{\nu+r}^{-\mu}(-t)(-1)^{r} \frac{\nu+r+\frac{1}{2}}{2 \cos \nu \pi} \int_{-c}^{c} g(x) P_{\nu+r}^{\mu}(x) d x\right) d t \\
\int_{-c}^{c} f(t)\left(\sum_{r=-n}^{n-1} b_{r} P_{\nu+r}^{-\mu}(-t)\right) d t \\
\text { where } b_{r}=(-1)^{r} \frac{\nu+r+\frac{1}{2}}{2 \cos \nu \pi} \int_{-c}^{c} g(x) P_{\nu+r}^{\mu}(x) d x
\end{gathered}
$$

So

$$
\begin{aligned}
\lim _{n \rightarrow \infty} \sum_{r=-n}^{n-1} a_{r} \int_{-c}^{c} P_{\nu+r}^{\mu}(x) g(x) d x=\lim _{n \rightarrow \infty} \int_{-c}^{c} f(t) \sum_{r=-n}^{n-1} b_{r} P_{\nu+r}^{-\mu}(-t) d t \\
\quad=\int_{-c}^{c} f(t) \lim _{n \rightarrow \infty} \sum_{r=-n}^{n-1} b_{r} P_{\nu+r}^{-\mu}(-t) d t \\
=\int_{-c}^{c} f(t) g(t) d t
\end{aligned}
$$

the steps (3) and (4) requiring justification. For (3) it is sufficient that $\sum_{r=-n}^{n-1} b_{r} P_{\nu+r}^{-\mu}(-t)$ be boundedly convergent as $n \rightarrow \infty$ and for (4) that $\sum_{r=-\infty}^{\infty} b_{r} \cdot P_{\nu+r}^{-\mu}(-t)=g(t)$ for almost all $t \in(-c, c)$.

The theorems actually proved in this paper are similar to Proposition 1, but differ in certain features. The main tasks are justifications like those of (3) and (4) above.

The restriction of the integrals to $(-c, c)$ instead of $(-1,1)$ is made because integration right to the singular points \pm 1 of the Legendre functions is not likely to be manageable. The Legendre functions involved are defined in terms of Gauss's hypergeometric function $F$ as in [1] (3.4(6)):

$$
P_{\nu}^{\mu}(x)=\frac{1}{\Gamma(1-\mu)}\left(\frac{1+x}{1-x}\right)^{\frac{1}{2} \mu} F\left(\begin{array}{c}
-\nu, 1+\nu ; \\
1-\mu ;
\end{array} \frac{1-x}{2}\right)
$$

for $-1<x<1$, except that $\mathrm{P}$, instead of $P$, is used in [1] for this particular function while $P$ is used for a different function.

The theorems can be adapted to any subinterval $(a, b)$ of $(-c, c)$ simply by defining $g$ to be zero outside $(a, b)$. 
Definition 1 (The Dini condition). $g$ is Dini at $x$ means that

$$
\frac{g(t)-g(x)}{t-x} \in L(x-\delta, x+\delta)
$$

for some $\delta>0$. All differentiable and all Lipschitz functions are Dini at all points concerned.

Lemma 1. If $0<c<1, g \in L(-c, c), g$ is Dini at almost all $x$ in $(-c, c)$, $-1 / 2<\mu<1 / 2$ and $-1 / 2<\nu<1 / 2$, then (as required for (4) above)

$$
g(x)=\sum_{r=-\infty}^{\infty} b_{r} P_{\nu+r}^{-\mu}(-x)
$$

for almost all $x \in(-c, c)$; $b_{r}$ being defined as in (2).

Proof. This comes from [2] (Theorem 8) by replacing its $\mu$ by $-\mu$, its $f(x)$ by $g(-x)$, and defining $g$ as zero outside $(-c, c)$; these replacements turn its $a_{r}$ into

$$
(-1)^{r} \frac{\nu+r+\frac{1}{2}}{2 \cos \nu \pi} \int_{-c}^{c} g(-t) P_{\nu+r}^{\mu}(-t) d t=b_{r}
$$

as in (2). The conclusion of [2] (Theorem 8) then gives

$$
g(-x)=\sum_{r=-\infty}^{\infty} b_{r} P_{\nu+r}^{-\mu}(x)
$$

at almost all $-x \in(-c, c)$; this is equivalent to the required result.

Lemma 2. If $0<c<1, g \in L(-c, c), g$ vanishes outside $(-c, c), \gamma=\arccos c$, $\gamma^{\prime}=\pi-\gamma, \gamma \leq \theta \leq \gamma^{\prime}, h(\phi)=g(\cos \phi) \sqrt{\sin \phi}, b_{r}$ is as in (2),

$$
s_{n}(x)=\sum_{r=-n}^{n-1} b_{r} P_{\nu+r}^{-\mu}(-x)
$$

and

$$
\begin{gathered}
D(\nu ; t, x) \equiv D(\mu, \nu ; t, x) \equiv \\
\frac{1}{2}\left\{(\nu-\mu) P_{\nu}^{\mu}(t) P_{\nu-1}^{-\mu}(-x)+(\nu+\mu) P_{\nu-1}^{\mu}(t) P_{\nu}^{-\mu}(-x)\right\}
\end{gathered}
$$

then

$$
\begin{gathered}
s_{n}(\cos \theta)= \\
\frac{(-1)^{n}}{2 \cos \nu \pi} \int_{\gamma}^{\gamma^{\prime}} \frac{D(\nu+n ; \cos \phi, \cos \theta)-D(\nu-n ; \cos \phi, \cos \theta)}{\cos \theta-\cos \phi} h(\phi) \sqrt{\sin \phi} d \phi .
\end{gathered}
$$


This lemma is essentially (3.6) of [2] with changed notation and $g$ vanishing outside $(-c, c)$. It is the counterpart of Dirichlet's Integral in trigonometric Fourier series theory. Formula (6) is (2.1) of [2] with $x$ and $t$ interchanged.

Lemma 3. If $\gamma=\pi / 6, \gamma^{\prime}=5 \pi / 6, \gamma \leq \theta \leq \gamma^{\prime},-1 / 2<\mu<1 / 2$ and $\nu \geq 1$ then

$$
\sqrt{\frac{1}{2} \pi \sin \theta} P_{\nu}^{\mu}(\cos \theta)=\frac{\Gamma(\nu+\mu+1)}{\Gamma\left(\nu+\frac{3}{2}\right)}\left[\sin \left\{\left(\nu+\frac{1}{2}\right) \theta+\left(\mu+\frac{1}{2}\right) \frac{1}{2} \pi\right\}+\omega_{1}\right]
$$

where $\omega_{1} \equiv \omega_{1}(\mu, \nu ; \theta)$ is $O(1 / \nu)$ on $S_{1}=\left\{(\nu, \theta): \nu \geq 1\right.$ and $\left.\gamma \leq \theta \leq \gamma^{\prime}\right\}$; also $\omega_{1}$ has continuous derivative $\partial \omega_{1} / \partial \theta$ which is bounded on $S_{1}$.

Proof. Comparing the stated equation with 3.5(5) of [1], it is seen that

$$
\begin{gathered}
\omega_{1}(\mu, \nu ; \theta)= \\
\sum_{r=1}^{\infty} \frac{\left(\frac{1}{2}+\mu\right)_{r}\left(\frac{1}{2}-\mu\right)_{r}}{\left(\nu+\frac{3}{2}\right)_{r}} \frac{\left(-\frac{1}{2} \csc \theta\right)^{r}}{r !} \sin \left\{\left(\nu+r+\frac{1}{2}\right) \theta+\left(\mu+r+\frac{1}{2}\right) \frac{1}{2} \pi\right\} .
\end{gathered}
$$

The $r$ th term of this series has modulus dominated on $S_{1}$ by

$$
\begin{aligned}
& \frac{\left(\frac{1}{2}+\mu\right)_{r}\left(\frac{1}{2}-\mu\right)_{r}}{\left(\nu+\frac{3}{2}\right)_{r} r !}=\frac{\left(\frac{1}{2}+\mu\right)_{r}\left(\frac{1}{2}-\mu\right)_{r}}{\left(\nu+\frac{3}{2}\right)\left(\nu+\frac{5}{2}\right)_{r-1}} \frac{1}{r !} \leq \frac{1}{\nu+\frac{3}{2}} \frac{\left(\frac{1}{2}+\mu\right)_{r}\left(\frac{1}{2}-\mu\right)_{r}}{\left(\frac{7}{2}\right)_{r-1} r !} \\
& =\frac{1}{\nu+\frac{3}{2}} \frac{\Gamma\left(\frac{7}{2}\right)}{\Gamma\left(\frac{1}{2}+\mu\right) \Gamma\left(\frac{1}{2}-\mu\right)} \frac{\Gamma\left(\frac{1}{2}+\mu+r\right) \Gamma\left(\frac{1}{2}-\mu+r\right)}{\Gamma\left(\frac{5}{2}+r\right) \Gamma(1+r)}=O\left(\frac{1}{\nu} \frac{1}{r^{5 / 2}}\right)
\end{aligned}
$$

where $O$ is independent of $(\nu, \theta)$ in $S_{1}$. In particular by Weierstrass's test the series (7) is uniformly convergent on $\gamma \leq \theta \leq \gamma^{\prime}$; and its sum is $O(1 / \nu)$ on $S_{1}$, which is one of the required conclusions.

Formal differentiation of (7) with respect to $\theta$ gives the series

$$
\begin{gathered}
\frac{\cos \theta}{2 \sin ^{2} \theta} \sum_{r=1}^{\infty} \frac{\left(\frac{1}{2}+\mu\right)_{r}\left(\frac{1}{2}-\mu\right)_{r}}{\left(\nu+\frac{3}{2}\right)_{r}} \frac{\left(-\frac{1}{2} \csc \theta\right)^{r-1}}{(r-1) !} \sin \left\{\left(\nu+r+\frac{1}{2}\right) \theta+\left(\mu+r+\frac{1}{2}\right) \frac{1}{2} \pi\right\} \\
-\frac{1}{2 \sin \theta} \sum_{r=1}^{\infty} \frac{\left(\frac{1}{2}+\mu\right)_{r}\left(\frac{1}{2}-\mu\right)_{r}}{\left(\nu+\frac{3}{2}\right)_{r}} \frac{\left(-\frac{1}{2} \csc \theta\right)^{r-1}}{(r-1) !} \frac{\nu+r+\frac{1}{2}}{r} C_{r} \\
\text { where } C_{r}=\cos \left\{\left(\nu+r+\frac{1}{2}\right) \theta+\left(\mu+r+\frac{1}{2}\right) \frac{1}{2} \pi\right\}
\end{gathered}
$$

Since

$$
\frac{\nu+r+\frac{1}{2}}{r}=1+\frac{\nu+\frac{1}{2}}{r} \leq \nu+\frac{3}{2}
$$


the $r$ th terms of both series in (8) have modulus dominated on $S_{1}$ by

$$
\begin{gathered}
\sqrt{3} \frac{\left(\frac{1}{2}+\mu\right)_{r}\left(\frac{1}{2}-\mu\right)_{r}}{\left(\nu+\frac{5}{2}\right)_{r-1}(r-1) !} \leq \sqrt{3} \frac{\left(\frac{1}{2}+\mu\right)_{r}\left(\frac{1}{2}-\mu\right)_{r}}{\left(\frac{7}{2}\right)_{r-1} \Gamma(r)}= \\
\sqrt{3} \frac{\Gamma\left(\frac{7}{2}\right)}{\Gamma\left(\frac{1}{2}+\mu\right) \Gamma\left(\frac{1}{2}-\mu\right)} \frac{\Gamma\left(\frac{1}{2}+\mu+r\right) \Gamma\left(\frac{1}{2}-\mu+r\right)}{\Gamma\left(\frac{5}{2}+r\right) \Gamma(r)}=O\left(\frac{1}{r^{3 / 2}}\right),
\end{gathered}
$$

where $O$ is independent of $(\nu, \theta)$ in $S_{1}$. So by Weierstrass's test again the series (8) are uniformly convergent on $\gamma \leq \theta \leq \gamma^{\prime}$; and since their terms are also continuous there, their combined sum is continuous and is the derivative of the sum of (7). Thus $\omega_{1}$ has continuous derivative $\partial \omega_{1} / \partial \theta$ in $\gamma \leq \theta \leq \gamma^{\prime}$. Further, that combined sum is bounded on $S_{1}$ because of the dominance (9); this gives the last conclusion, that $\partial \omega_{1} / \partial \theta$ is bounded on $S_{1}$.

Lemma 4. If $\gamma=\pi / 6, \gamma^{\prime}=5 \pi / 6, \gamma \leq \theta \leq \gamma^{\prime}, \gamma \leq \phi \leq \gamma^{\prime}, \theta^{\prime}=\pi-\theta$, $-1 / 2<\mu<1 / 2$ and $\nu \geq 2$, then $D$, defined at (6) in Lemma 2, satisfies

$$
\begin{gathered}
\pi \sqrt{\sin \theta \sin \phi} D(\nu ; \cos \phi, \cos \theta)= \\
\sin \nu\left(\phi+\theta^{\prime}\right) \cos \frac{1}{2}\left(\phi-\theta^{\prime}\right)+\cos \left\{\nu\left(\phi-\theta^{\prime}\right)+\mu \pi\right\} \cos \frac{1}{2}\left(\phi+\theta^{\prime}\right)+\omega_{2},
\end{gathered}
$$

where $\omega_{2} \equiv \omega_{2}(\mu, \nu ; \theta, \phi)$ is $O(1 / \nu)$ on $S_{2}=\left\{(\nu, \theta, \phi): \nu \geq 2, \gamma \leq \theta \leq \gamma^{\prime}\right.$ and $\left.\gamma \leq \phi \leq \gamma^{\prime}\right\}$; also $\omega_{2}$ has continuous derivatives $\partial \omega_{2} / \partial \theta$ and $\partial \omega_{2} / \partial \phi$, which are bounded on $S_{2}$.

Proof. By (6) and Lemma 3, the expression in question is equal to

$$
\begin{gathered}
\frac{1}{2} \pi \sqrt{\sin \theta \sin \phi} \times \\
\left\{(\nu-\mu) P_{\nu}^{\mu}(\cos \phi) P_{\nu-1}^{-\mu}\left(\cos \theta^{\prime}\right)+(\nu+\mu) P_{\nu-1}^{\mu}(\cos \phi) P_{\nu}^{-\mu}\left(\cos \theta^{\prime}\right)\right\} \\
=(\nu-\mu) \frac{\Gamma(\nu+\mu+1)}{\Gamma\left(\nu+\frac{3}{2}\right)} \frac{\Gamma(\nu-\mu)}{\Gamma\left(\nu+\frac{1}{2}\right)}\left[\sin \left\{\left(\nu+\frac{1}{2}\right) \phi+\left(\mu+\frac{1}{2}\right) \frac{1}{2} \pi\right\}+\omega_{1}(\mu, \nu ; \phi)\right] \\
\times\left[\sin \left\{\left(\nu-\frac{1}{2}\right) \theta^{\prime}-\left(\mu-\frac{1}{2}\right) \frac{1}{2} \pi\right\}+\omega_{1}\left(-\mu, \nu-1 ; \theta^{\prime}\right)\right] \\
+(\nu+\mu) \frac{\Gamma(\nu+\mu)}{\Gamma\left(\nu+\frac{1}{2}\right)} \frac{\Gamma(\nu-\mu+1)}{\Gamma\left(\nu+\frac{3}{2}\right)}\left[\sin \left\{\left(\nu-\frac{1}{2}\right) \phi+\left(\mu+\frac{1}{2}\right) \frac{1}{2} \pi\right\}+\omega_{1}(\mu, \nu-1 ; \phi)\right] \\
\times\left[\sin \left\{\left(\nu+\frac{1}{2}\right) \theta^{\prime}-\left(\mu-\frac{1}{2}\right) \frac{1}{2} \pi\right\}+\omega_{1}\left(-\mu, \nu ; \theta^{\prime}\right)\right] \\
=\frac{\Gamma(\nu+\mu+1)}{\Gamma\left(\nu+\frac{3}{2}\right)} \frac{\Gamma(\nu-\mu+1)}{\Gamma\left(\nu+\frac{1}{2}\right)} \frac{1}{2} \times
\end{gathered}
$$




$$
\begin{array}{r}
{\left[\cos \left\{\nu\left(\phi-\theta^{\prime}\right)+\frac{1}{2}\left(\phi+\theta^{\prime}\right)+\mu \pi\right\}-\cos \left\{\nu\left(\phi+\theta^{\prime}\right)+\frac{1}{2}\left(\phi-\theta^{\prime}\right)+\frac{1}{2} \pi\right\}+\right.} \\
\left.\cos \left\{\nu\left(\phi-\theta^{\prime}\right)-\frac{1}{2}\left(\phi+\theta^{\prime}\right)+\mu \pi\right\}-\cos \left\{\nu\left(\phi+\theta^{\prime}\right)-\frac{1}{2}\left(\phi-\theta^{\prime}\right)+\frac{1}{2} \pi\right\}+2 \omega\right],
\end{array}
$$

where $\omega=\omega(\mu, \nu ; \theta, \phi)$ is equal to

$$
\begin{gathered}
\omega_{1}(\mu, \nu ; \phi) \sin \left\{\left(\nu-\frac{1}{2}\right) \theta^{\prime}-\left(\mu-\frac{1}{2}\right) \frac{1}{2} \pi\right\}+ \\
\omega_{1}\left(-\mu, \nu-1 ; \theta^{\prime}\right) \sin \left\{\left(\nu+\frac{1}{2}\right) \phi+\left(\mu+\frac{1}{2}\right) \frac{1}{2} \pi\right\}+\omega_{1}(\mu, \nu ; \phi) \omega_{1}\left(-\mu, \nu-1 ; \theta^{\prime}\right) \\
+\omega_{1}(\mu, \nu-1 ; \phi) \sin \left\{\left(\nu+\frac{1}{2}\right) \theta^{\prime}-\left(\mu-\frac{1}{2}\right) \frac{1}{2} \pi\right\}+ \\
\omega_{1}\left(-\mu, \nu ; \theta^{\prime}\right) \sin \left\{\left(\nu-\frac{1}{2}\right) \phi+\left(\mu+\frac{1}{2}\right) \frac{1}{2} \pi\right\}+\omega_{1}(\mu, \nu-1 ; \phi) \omega_{1}\left(-\mu, \nu, \theta^{\prime}\right)
\end{gathered}
$$

By Lemma 3 this $\omega$ is $O(1 / \nu)$ on $S_{2}$, and it has continuous derivatives $\partial \omega / \partial \theta$ and $\partial \omega / \partial \phi$ thereon. Further the term in $\partial \omega / \partial \theta$ corresponding to the first line of $(12)$ is

$$
-\omega_{1}(\mu, \nu ; \phi)\left(\nu-\frac{1}{2}\right) \cos \left\{\left(\nu-\frac{1}{2}\right) \theta^{\prime}-\left(\mu-\frac{1}{2}\right) \frac{1}{2} \pi\right\} ;
$$

that is, bounded on $S_{2}$ by Lemma 3 . Similarly the other terms in (12); so that $\partial \omega / \partial \theta$ is bounded on $S_{2}$. Equally $\partial \omega / \partial \phi$ is bounded on $S_{2}$.

By 1.18(4) of [1] and addition theorems, (11) is equal to

$$
(1+\rho)\left[\cos \left\{\nu\left(\phi-\theta^{\prime}\right)+\mu \pi\right\} \cos \frac{1}{2}\left(\phi+\theta^{\prime}\right)-\cos \left\{\nu\left(\phi+\theta^{\prime}\right)+\frac{1}{2} \pi\right\} \cos \frac{1}{2}\left(\phi-\theta^{\prime}\right)+\omega\right]
$$

where $\rho=O(1 / \nu)$ and $\rho$ is independent of $\theta$ and $\phi$. This expression is equal to

$$
\cos \left\{\nu\left(\phi-\theta^{\prime}\right)+\mu \pi\right\} \cos \frac{1}{2}\left(\phi+\theta^{\prime}\right)+\sin \nu\left(\phi+\theta^{\prime}\right) \cos \frac{1}{2}\left(\phi-\theta^{\prime}\right)+\omega_{2},
$$

the required formula, with

$$
\begin{gathered}
\omega_{2} \equiv \omega_{2}(\mu, \nu ; \theta, \phi)= \\
\left.\rho\left[\cos \nu\left(\phi-\theta^{\prime}\right)+\mu \pi\right\} \cos \frac{1}{2}\left(\phi+\theta^{\prime}\right)+\sin \nu\left(\phi+\theta^{\prime}\right) \cos \frac{1}{2}\left(\phi-\theta^{\prime}\right)\right]+(1+\rho) \omega .
\end{gathered}
$$

It is clear from the above properties of $\rho$ and $\omega$ that $\omega_{2}=O(1 / \nu)$ on $S_{2}$, and that $\omega_{2}$ has continuous derivatives $\partial \omega_{2} / \partial \theta$ and $\partial \omega_{2} / \partial \phi$; and also that these derivatives are bounded on $S_{2}$ since $\rho \nu=O(1)$. This completes the proof of Lemma 4. 
Lemma 5. If the hypothesis of Lemma 4 hold, except that $\nu$ is now fixed with $-1 / 2<\nu<1 / 2$ and $n$ is an integer greater than two $(\nu+n$ replacing the previous $\nu$ ) then $s_{n}(x)$, defined at (5) in Lemma 2, is given by

$$
\begin{array}{r}
s_{n}(\cos \theta)=\frac{\sec \nu \pi}{2 \pi \sqrt{(\sin \theta)}}\left[\int_{\gamma}^{\gamma^{\prime}} \frac{\sin n(\phi-\theta)}{\sin \frac{1}{2}(\phi-\theta)} \cos \nu\left(\phi+\theta^{\prime}\right) h(\phi) d \phi-\right. \\
\left.\int_{\gamma}^{\gamma^{\prime}} \frac{\cos \{n(\phi+\theta)+\mu \pi\}}{\sin \frac{1}{2}(\phi+\theta)} \cos \nu\left(\phi-\theta^{\prime}\right) h(\phi) d \phi+\int_{\gamma}^{\gamma^{\prime}} \frac{\Omega(n ; \theta, \phi)}{\cos \theta-\cos \phi} h(\phi) d \phi\right],
\end{array}
$$

where $\Omega(n ; \theta, \phi) \equiv \Omega(\mu, \nu, n ; \theta, \phi)$ is $O(1 / n)$ on $S_{3}=\{(n, \theta, \phi): n \geq 3$, $\gamma \leq \theta \leq \gamma^{\prime}$ and $\left.\gamma \leq \phi \leq \gamma^{\prime}\right\}$; also $\Omega$ has continuous derivatives $\partial \Omega / \partial \theta$ and $\partial \Omega / \partial \phi$, which are bounded on $S_{3}$.

Proof. By (6) and 3.4(7) of [1],

$$
\begin{aligned}
& D(-\lambda ; t, x)=\frac{1}{2}\left\{(-\lambda-\mu) P_{-\lambda}^{\mu}(t) P_{-\lambda-1}^{-\mu}(-x)+(-\lambda+\mu) P_{-\lambda-1}^{\mu}(t) P_{-\lambda}^{-\mu}(-x)\right\} \\
& =\frac{1}{2}\left\{-(\lambda+\mu) P_{\lambda-1}^{\mu}(t) P_{\lambda}^{-\mu}(-x)-(\lambda-\mu) P_{\lambda}^{\mu}(t) P_{\lambda-1}^{-\mu}(-x)\right\}=-D(\lambda ; t, x) ;
\end{aligned}
$$

whence

$$
-D(\nu-n ; \cos \phi, \cos \theta)=D(n-\nu ; \cos \phi, \cos \theta) .
$$

Replacing $\nu$ in Lemma 4 by $n+\nu$ and $n-\nu$, and using (14),

$$
\begin{gathered}
\frac{1}{2} \pi \sqrt{\sin \theta \sin \phi}\{D(\nu+n ; \cos \phi, \cos \theta)-D(\nu-n ; \cos \phi, \cos \theta)\}= \\
\frac{1}{2}\left[\sin \left\{(n+\nu)\left(\phi+\theta^{\prime}\right)\right\} \sin \frac{1}{2}(\phi+\theta)-\cos \left\{(n+\nu)\left(\phi-\theta^{\prime}\right)+\mu \pi\right\} \sin \frac{1}{2}(\phi-\theta)\right. \\
+\omega_{2}(\mu, n+\nu ; \theta, \phi)+\sin \left\{(n-\nu)\left(\phi+\theta^{\prime}\right\} \sin \frac{1}{2}(\phi+\theta)-\right. \\
\left.\cos \left\{(n-\nu)\left(\phi-\theta^{\prime}\right)+\mu \pi\right\} \sin \frac{1}{2}(\phi-\theta)+\omega_{2}(\mu, n-\nu ; \theta, \phi)\right]= \\
\sin n\left(\phi+\theta^{\prime}\right) \cos \nu\left(\phi+\theta^{\prime}\right) \sin \frac{1}{2}(\phi+\theta)-\cos \left\{n\left(\phi-\theta^{\prime}\right)+\mu \pi\right\} \cos \nu\left(\phi-\theta^{\prime}\right) \sin \frac{1}{2}(\phi-\theta) \\
+\frac{1}{2}(-1)^{n} \Omega(n ; \theta, \phi), \\
\text { where } \Omega(n ; \theta, \phi)=(-1)^{n}\left\{\omega_{2}(\mu, n+\nu ; \theta, \phi)+\omega_{2}(\mu, n-\nu ; \theta, \phi)\right\} .
\end{gathered}
$$

By Lemma $4, \Omega(n ; \theta, \phi)$ is $O(1 / n)$ on $S_{3}$, it has continuous derivatives $\partial \Omega / \partial \theta$ and $\partial \Omega / \partial \phi$, and these are bounded on $S_{3}$. By Lemma 2 , and (14) and (15), provided the integrals exist,

$$
s_{n}(\cos \theta)=\frac{(-1)^{n} \sec \nu \pi}{2 \pi \sqrt{(\sin \theta)}}\left[\int_{\gamma}^{\gamma^{\prime}} \frac{\sin n\left(\phi+\theta^{\prime}\right) \cos \nu\left(\phi+\theta^{\prime}\right)}{\sin \frac{1}{2}(\phi-\theta)} h(\phi) d \phi-\right.
$$




$$
\left.\int_{\gamma}^{\gamma^{\prime}} \frac{\cos \left\{n\left(\phi-\theta^{\prime}\right)+\mu \pi\right\} \cos \nu\left(\phi-\theta^{\prime}\right)}{\sin \frac{1}{2}(\phi+\theta)} h(\phi) d \phi+(-1)^{n} \int_{\gamma}^{\gamma^{\prime}} \frac{\Omega(n ; \theta, \phi)}{\cos \theta-\cos \phi} h(\phi) d \phi\right]
$$

this is equivalent to the stated result by $\theta^{\prime}=\pi-\theta$, subject to existence of the integrals.

Of the three integrals in (13), the first exists because $h \in L\left(\gamma, \gamma^{\prime}\right)$ and $h$ is multiplied by a continuous function; the second exists for the same reason, since $0<\gamma \leq \frac{1}{2}(\phi+\theta) \leq \gamma^{\prime}<\pi$ so that the denominator $\sin \frac{1}{2}(\phi+\theta)$ does not vanish. The third integral in (13) exists by linearity, despite the denominator; because its integrand is a linear combination of the integrand of $s_{n}(\cos \theta)$ in Lemma 2 and the first two integrands in (13), and these three integrands are all integrable.

Lemma 6. If $0<\gamma<\pi / 2$ and $\gamma \leq \theta \leq \gamma^{\prime}$, then

$$
\int_{\gamma}^{\gamma^{\prime}}|\log | \cos \phi-\cos \theta|| d \phi<\frac{2 \cos \gamma \log (4 e)+\pi e^{-1}}{\sin \gamma},
$$

a bound independent of $\theta$.

Proof. The integral is equal to

$$
\begin{gathered}
\quad \int_{\gamma}^{\gamma^{\prime}}|\log | \frac{\cos \phi-\cos \theta}{2}|+\log 2| d \phi \\
\leq \int_{\gamma}^{\gamma^{\prime}}\left(\log \left|\frac{2}{\cos \phi-\cos \theta}\right|+\log 2\right) d \phi \\
\leq \int_{\gamma}^{\gamma^{\prime}}\{2 \log 2-\log |\cos \phi-\cos \theta|\} \frac{\sin \phi}{\sin \gamma} d \phi \\
=\csc \gamma[-2(\log 2) \cos \phi+(\cos \phi-\cos \theta) \log |\cos \phi-\cos \theta|-\cos \phi]_{\phi=\gamma}^{\gamma^{\prime}} \\
\leq \csc \gamma\left\{2(2 \log 2+1) \cos \gamma+\left(\gamma^{\prime}-\gamma\right) e^{-1}\right\} \\
<\csc \gamma\left\{2 \log (4 e) \cos \gamma+\pi e^{-1}\right\}
\end{gathered}
$$

Lemma 7. If $0<\gamma<\pi / 2$ and $h$ has bounded variation on $\left[\gamma, \gamma^{\prime}\right]$, then

$$
\int_{\gamma}^{\gamma^{\prime}} \cos \nu\left(\phi+\theta^{\prime}\right) \frac{\sin n(\phi-\theta)}{\sin \frac{1}{2}(\phi-\theta)} h(\phi) d \phi
$$

is bounded on the set $S_{4}=\left\{(n, \theta): n \geq 1\right.$ and $\left.\gamma \leq \theta \leq \gamma^{\prime}\right\}$. 
Proof. (i) Omitting the factor $h(\phi)$ for the moment, the integrand is equal to

$$
\begin{gathered}
\{\cos \nu \pi \cos \nu(\phi-\theta)-\sin \nu \pi \sin \nu(\phi-\theta)\} \frac{\sin n(\phi-\theta)}{\sin \frac{1}{2}(\phi-\theta)}= \\
\cos \nu \pi \frac{\sin (n+\nu)(\phi-\theta)+\sin (n-\nu)(\phi-\theta)}{2 \sin \frac{1}{2}(\phi-\theta)}-\sin \nu \pi \frac{\sin \nu(\phi-\theta)}{\sin \frac{1}{2}(\phi-\theta)} \sin n(\phi-\theta) .
\end{gathered}
$$

The latter term is bounded independently of $n, \theta$ and $\phi$ since, for $\gamma \leq \theta \leq \gamma^{\prime}$ and $\gamma \leq \phi \leq \gamma^{\prime},|\phi-\theta| \leq \gamma^{\prime}-\gamma=\pi-2 \gamma<\pi$. Also $h$ is bounded. So the part of the integral in question corresponding to the latter term is bounded on $S_{4}$. It is therefore sufficient to prove boundedness of

$$
\int_{\gamma}^{\gamma^{\prime}} \frac{\sin (n \pm \nu)(\phi-\theta)}{\sin \frac{1}{2}(\phi-\theta)} h(\phi) d \phi .
$$

Since $\nu$ is unrestricted we need only consider the plus case.

(ii) For $-\pi<\chi<\pi$ let

$$
\begin{gathered}
F_{n}(\chi)=\int_{0}^{\chi} \frac{\sin (n+\nu) \psi}{\sin \frac{1}{2} \psi} d \psi= \\
\int_{0}^{\chi}\left(\frac{1}{\sin \frac{1}{2} \psi}-\frac{1}{\frac{1}{2} \psi}\right) \sin (n+\nu) \psi d \psi+\int_{0}^{\chi} \frac{\sin (n+\nu) \psi}{\frac{1}{2} \psi} d \psi .
\end{gathered}
$$

For $0<\chi<\pi$ the first factor in the integrand of the former integral in (17) is positive and less than

$$
\frac{1}{\sin \frac{1}{2} \psi}-\frac{1}{\tan \frac{1}{2} \psi}=\frac{1-\cos \frac{1}{2} \psi}{\sin \frac{1}{2} \psi}=\frac{\sin \frac{1}{2} \psi}{1+\cos \frac{1}{2} \psi}<\frac{\sin \frac{1}{2} \chi}{1+\cos \frac{1}{2} \chi}<\sin \frac{1}{2} \chi<1 ;
$$

so the former integral in (17) has modulus less than $\pi$. Similarly for $-\pi<$ $\chi<0$. It is standard that the latter integral in (17) is a bounded function of $n$ and $\chi$. Thus $F_{n}(\chi)$ is also a bounded function of $n$ and $\chi$ on $-\pi<\chi<\pi$.

(iii) The integral (16) (with plus) is equal to

$$
\begin{gathered}
\int_{\gamma-\theta}^{\gamma^{\prime}-\theta} \frac{\sin (n+\nu) \psi}{\sin \frac{1}{2} \psi} h(\theta+\psi) d \psi=F_{n}\left(\gamma^{\prime}-\theta\right) h\left(\gamma^{\prime}\right)-F_{n}(\gamma-\theta) h(\gamma)- \\
\int_{\gamma-\theta}^{\gamma^{\prime}-\theta} F_{n}(\psi) d_{\psi} h(\theta+\psi)=F_{n}\left(\gamma^{\prime}-\theta\right) h\left(\gamma^{\prime}\right)-F_{n}(\gamma-\theta) h(\gamma)-\int_{\gamma}^{\gamma^{\prime}} F_{n}(\phi-\theta) d h(\phi) .
\end{gathered}
$$

All three of these terms are bounded functions of $(n, \theta)$ on $S_{4}$ by (ii), remembering that $h$ has bounded variation on $\left[\gamma, \gamma^{\prime}\right]$. This completes the proof. 
Lemma 8. If the hypotheses of Lemma 5 hold, $x=\cos \theta$ and $c=\cos \gamma=$ $\sqrt{3} / 2, g$ has bounded variation on $[-c, c]$ and for almost all $x$

$$
\int_{-c}^{c}\left|\frac{g(t)-g(x)}{t-x}\right| d t<\infty
$$

then $h(\phi)$ and $\Omega(n ; \theta, \phi)$, occurring in Lemma 2 and 5 , satisfy, for almost all $x$,

$\int_{\gamma}^{\gamma^{\prime}} \frac{\Omega(n ; \theta, \phi)}{\cos \phi-\cos \theta} h(\phi) d \phi=O\left(\int_{-c}^{c}\left|\frac{g(t)-g(x)}{t-x}\right| d t\right)+O\left(\log \frac{1}{c^{2}-x^{2}}\right)$

on the set $S_{5}=\{(n, x): n \geq 3$ and $-c<x<c\}$.

Proof. (i) For $\theta$ and $\phi$ in $\left[\gamma, \gamma^{\prime}\right]$, remembering that $h(\phi)=g(\cos \phi) \sqrt{\sin \phi}$,

$$
\begin{aligned}
& \frac{h(\phi)-h(\theta)}{\cos \phi-\cos \theta}=g(\cos \phi) \frac{\sqrt{\sin \phi}-\sqrt{\sin \theta}}{\cos \phi-\cos \theta}+\sqrt{\sin \theta} \frac{g(\cos \phi)-g(\cos \theta)}{\cos \phi-\cos \theta} \\
& =-\frac{g(\cos \phi)}{\sqrt{\sin \phi}+\sqrt{\sin \theta}} \cot \frac{1}{2}(\phi+\theta)+\frac{\sqrt{\sin \theta}}{\sin \phi} \frac{g(\cos \phi)-g(\cos \theta)}{\cos \phi-\cos \theta} \sin \phi .
\end{aligned}
$$

Since $\sin \phi \geq \sin \gamma, \sin \theta \geq \sin \gamma, \sin \frac{1}{2}(\phi+\theta) \geq \sin \gamma=\frac{1}{2}$ and $g \in L(-c, c)$,

$$
\begin{gathered}
\int_{\gamma}^{\gamma^{\prime}}\left|\frac{h(\phi)-h(\theta)}{\cos \phi-\cos \theta}\right| d \phi \leq \\
\frac{1}{2(\sin \gamma)^{5 / 2}} \int_{\gamma}^{\gamma^{\prime}}|g(\cos \phi)| \sin \phi d \phi+\frac{1}{\sin \gamma} \int_{\gamma}^{\gamma^{\prime}}\left|\frac{g(\cos \phi)-g(\cos \theta)}{\cos \phi-\cos \theta}\right| \sin \phi d \phi \\
=2 \sqrt{2} \int_{-c}^{c}|g(t)| d t+2 \int_{-c}^{c}\left|\frac{g(t)-g(x)}{t-x}\right| d t
\end{gathered}
$$

By hypothesis (19) is finite for almost all $x \in(-c, c)$.

(ii) Provided that two of the following three integrals exist,

$$
\begin{gathered}
\int_{\gamma}^{\gamma^{\prime}} \frac{\Omega(n ; \theta, \phi)}{\cos \phi-\cos \theta} h(\phi) d \phi= \\
\int_{\gamma}^{\gamma^{\prime}} \Omega(n ; \theta, \phi) \frac{h(\phi)-h(\theta)}{\cos \phi-\cos \theta} d \phi+h(\theta) \int_{\gamma}^{\gamma^{\prime}} \frac{\Omega(n ; \theta, \phi)}{\cos \phi-\cos \theta} d \phi .
\end{gathered}
$$


The last paragraph of the proof of Lemma 5 shows that the integral on the left of (20) exists. The first integral on the right exists for almost all $\theta$, by (19) and the boundedness of $\Omega$ as a function of $\phi$, which follows from Lemma 5. Thus (20), including the existence of the integrals in it, is established for almost all $\theta$.

(iii) The last integral in (20), being Lebesgue as shown in (ii), is equal to the limit as $\eta \rightarrow 0+$ of

$$
\begin{gathered}
\left(\int_{\gamma}^{\theta-\eta}+\int_{\theta+\eta}^{\gamma^{\prime}}\right) \frac{\Omega(n ; \theta, \phi)}{\sin \phi} \frac{\sin \phi}{\cos \phi-\cos \theta} d \phi= \\
-\left[\frac{\Omega(n ; \theta, \phi)}{\sin \phi} \log |\cos \phi-\cos \theta|\right]_{\phi=\gamma}^{\theta-\eta}-\left[\frac{\Omega(n ; \theta, \phi)}{\sin \phi} \log |\cos \phi-\cos \theta|\right]_{\phi=\theta+\eta}^{\gamma^{\prime}} \\
+\left(\int_{\gamma}^{\theta-\eta}+\int_{\theta+\eta}^{\gamma^{\prime}}\right)\left(\frac{1}{\sin \phi} \frac{\partial \Omega}{\partial \phi}-\frac{\cos \phi}{\sin ^{2} \phi} \Omega\right) \log |\cos \phi-\cos \theta| d \phi
\end{gathered}
$$

integration by parts being valid since $\partial \Omega / \partial \phi$ is continuous by Lemma 5 .

The function $\omega(\phi)=\Omega(n ; \theta, \phi) / \sin \phi$ has continuous derivative in $\gamma \leq \phi \leq$ $\gamma^{\prime}$. The part of line (21) involving $\eta$ is therefore equal to

$$
\begin{gathered}
-\omega(\theta-\eta) \log |\cos (\theta-\eta)-\cos \theta|+\omega(\theta+\eta) \log |\cos (\theta+\eta)-\cos \theta|= \\
-\{\omega(\theta)+O(\eta)\} \log \{\cos (\theta-\eta)-\cos \theta\}+\{\omega(\theta)+O(\eta)\} \log \{\cos \theta-\cos (\theta+\eta)\} \\
=\omega(\theta) \log \frac{\cos \theta-\cos (\theta+\eta)}{\cos (\theta-\eta)-\cos \theta}+O(\eta \log \eta) \rightarrow 0 \text { as } \eta \rightarrow 0+.
\end{gathered}
$$

So by (21) and (22) the last integral in (20) is equal, for almost all $\theta$, to

$$
\begin{array}{r}
\frac{\Omega(n ; \theta, \gamma)}{\sin \gamma} \log |\cos \gamma-\cos \theta|-\frac{\Omega\left(n ; \theta, \gamma^{\prime}\right)}{\sin \gamma^{\prime}} \log \left|\cos \gamma^{\prime}-\cos \theta\right| \\
+\int_{\gamma}^{\gamma^{\prime}}\left(\frac{1}{\sin \phi} \frac{\partial \Omega}{\partial \phi}(n ; \theta, \phi)-\frac{\cos \phi}{\sin ^{2} \phi} \Omega(n ; \theta, \phi)\right) \log |\cos \phi-\cos \theta| d \phi .
\end{array}
$$

Line (24) is bounded on $S_{5}$, by Lemmas 5 and 6 . Lemma 5 also shows that line $(23)$ is, on $S_{5}$,

$$
O\left(\frac{1}{n} \log (\cos \gamma-\cos \theta)\right)+O\left(\frac{1}{n} \log \left(\cos \theta-\cos \gamma^{\prime}\right)\right)
$$




$$
\begin{gathered}
=O\left(\log \frac{2}{\cos \gamma-\cos \theta}+\log 2\right)+O\left(\log \frac{2}{\cos \theta+\cos \gamma}+\log 2\right) \\
=O\left(\log \frac{4}{\cos ^{2} \gamma-\cos ^{2} \theta}\right)+O(1) \\
=O\left(\log \frac{1}{c^{2}-x^{2}}\right)+O(1)=O\left(\log \frac{1}{c^{2}-x^{2}}\right) .
\end{gathered}
$$

This, together with (20) and (19), establishes the stated result (18), thus completing the proof of Lemma 8.

Theorem 1. Let $-1 / 2<\mu<1 / 2,-1 / 2<\nu<1 / 2, c=\sqrt{3} / 2 \simeq 0.866, g$ have bounded variation on $[-c, c]$ and

$$
\int_{-c}^{c} d x \int_{-c}^{c}\left|\frac{g(t)-g(x)}{t-x}\right| d t<\infty
$$

If

$$
b_{r}=(-1)^{r} \frac{\nu+r+\frac{1}{2}}{2 \cos \nu \pi} \int_{-c}^{c} g(t) P_{\nu+r}^{\mu}(t) d t \text { and } s_{n}(x)=\sum_{r=-n}^{n-1} b_{r} P_{\nu+r}^{-\mu}(-x)
$$

then $s_{n}(x)$ converges to $g(x)$ dominatedly on $-c \leq x \leq c$ as $n \rightarrow \infty$.

Proof. The inner integral in (25) is finite almost everywhere in $-c \leq x \leq c$; consequently $g$ is Dini at those points. Lemma 1 now gives that $s_{n}(x) \rightarrow g(x)$ almost everywhere as $n \rightarrow \infty$. It only remains to prove that this convergence is dominated.

By Lemma 5, and with its notation, $s_{n}(x)$ is equal to the sum of three terms multiplied by a bounded factor; and in those terms we have $h(\phi)=$ $g(\cos \phi) \sqrt{(\sin \phi)}$ and $\gamma \leq \phi \leq \gamma^{\prime}$, so that $h$ has bounded variation on $\left[\gamma, \gamma^{\prime}\right]$.

Of those three terms (integrals) in Lemma 5 , the first is bounded on $S_{4}$, by Lemma 7 . The second is obviously bounded since $\sin \frac{1}{2}(\phi+\theta) \geq \sin \gamma$ for all $\theta$ and $\phi$ involved, and $h(\phi)$ is bounded. Lemma 8 gives that the third term is

$$
O\left(\int_{-c}^{c}\left|\frac{g(t)-g(x)}{t-x}\right| d t\right)+O\left(\log \frac{1}{c^{2}-x^{2}}\right)
$$

and since the other two terms are bounded, $s_{n}(x)$ is dominated by just such an expression, which is a positive integrable function independent of $n$, by (25). 
Theorem 2. Let $-1 / 2<\mu<1 / 2,-1 / 2<\nu<1 / 2$ and $c=\sqrt{3} / 2$. Let $f$ be essentially bounded on $[-c, c]$ and zero outside that interval; and let

$$
a_{r}=(-1)^{r} \frac{\nu+r+\frac{1}{2}}{2 \cos \nu \pi} \int_{-c}^{c} f(t) P_{\nu+r}^{-\mu}(-t) d t .
$$

Let $g$ have bounded variation on $[-c, c]$,

$$
b_{r}=(-1)^{r} \frac{\nu+r+\frac{1}{2}}{2 \cos \nu \pi} \int_{-c}^{c} g(t) P_{\nu+r}^{\mu}(t) d t .
$$

and

$$
\int_{-c}^{c} d x \int_{-c}^{c}\left|\frac{g(t)-g(x)}{t-x}\right| d t<\infty .
$$

Then, even if there are values of $x$ such that

$$
f(x) \neq \sum_{-\infty}^{\infty} a_{r} P_{\nu+r}^{\mu}(x)
$$

or such that the series in (27) is divergent, it is nevertheless true that

$$
\int_{-c}^{c} f(x) g(x) d x=\sum_{-\infty}^{\infty} a_{r} \int_{-c}^{c} P_{\nu+r}^{\mu}(x) g(x) d x .
$$

Proof. The integrals on the right of (28) exist since $P_{\nu+r}^{\mu}(x)$ is continuous in $-c \leq x \leq c$ and $g(x)$ is bounded thereon. As in the equations preceding (2), and with the notation (26)

$$
\sum_{r=-n}^{n-1} a_{r} \int_{-c}^{c} P_{\nu+r}^{\mu}(x) g(x) d x=\int_{-c}^{c} f(t) s_{n}(t) d t \rightarrow \int_{-c}^{c} f(t) g(t) d t
$$

as $n \rightarrow \infty$; this limit process is valid because $s_{n}(t)$ converges dominatedly to $g(t)$ by Theorem 1 and $f(t)$ is essentially bounded, so that the whole integrand $f(t) s_{n}(t)$ also converges dominatedly. This proves Theorem 2 .

Corollary 1. If $-c \leq a<b \leq c$ then (compare (27) and (28))

$$
\int_{a}^{b} f(x) d x=\sum_{r=-\infty}^{\infty} a_{r} \int_{a}^{b} P_{\nu+r}^{\mu}(x) d x .
$$


Proof. In Theorem 2 take $g(x)=1$ in $a<x<b, g(x)=0$ elsewhere. Then $g$ is certainly of bounded variation, but we must verify (25). The double integral is equal to

$$
\begin{aligned}
& \int_{-c}^{c} d x \int_{-c}^{a}\left|\frac{g(x)}{t-x)}\right| d t+\int_{-c}^{c} d x \int_{a}^{b}\left|\frac{1-g(x)}{t-x}\right| d t+\int_{-c}^{c} d x \int_{b}^{c}\left|\frac{g(x)}{t-x}\right| d t \\
= & \int_{a}^{b} d x \int_{-c}^{a} \frac{d t}{x-t}+\int_{-c}^{a} d x \int_{a}^{b} \frac{d t}{t-x}+\int_{b}^{c} d x \int_{a}^{b} \frac{d t}{x-t}+\int_{a}^{b} d x \int_{b}^{c} \frac{d t}{t-x} \\
= & \int_{a}^{b} \log \frac{x+c}{x-a} d x+\int_{-c}^{a} \log \frac{b-x}{a-x} d x+\int_{b}^{c} \log \frac{x-a}{x-b} d x+\int_{a}^{b} \log \frac{c-x}{b-x} d x
\end{aligned}
$$

and these four integrals are all convergent.

Remark 1. The integrals in (28) can similarly be taken over $(a, b)$, any subinterval of $(-c, c)$, by replacing $g(x)$ by 0 outside $(a, b)$.

Lemma 9. Let $-1 / 2<\mu<1 / 2,-1 / 2<\nu<1 / 2$ and $c=\sqrt{3} / 2$. Let $g$ have bounded variation on $[-c, c]$, and

$$
\int_{-c}^{c}\left|\frac{g(t)-g(x)}{t-x}\right| d t=O\left(\log \frac{1}{c^{2}-x^{2}}\right)
$$

almost everywhere in $-c<x<c$. If $b_{r}$ and $s_{n}(x)$ are as in (26), then $s_{n}(x) / \log \left(c^{2}-x^{2}\right)$ converges boundedly on $-c<x<c$ to $g(x) / \log \left(c^{2}-x^{2}\right)$ as $n \rightarrow \infty$.

Proof. It is assured by (29) that $g$ is Dini at almost all $x$. So, as in the first paragraph of the proof of Theorem $1, s_{n}(x) \rightarrow g(x)$ almost everywhere as $n \rightarrow \infty$; thus

$$
\sigma_{n}(x) \equiv \frac{s_{n}(x)}{\log \left(c^{2}-x^{2}\right)} \rightarrow \frac{g(x)}{\log \left(c^{2}-x^{2}\right)}
$$

almost everywhere. Continuing as in the proof of Theorem $1, \sigma_{n}(\cos \theta)$ is, by Lemma 5 , equal to the sum of three terms, two of which are bounded on the set $S_{6}$ which we now define as $S_{4}$ omitting a certain null set of values of $\theta$ (see Lemma 7 for $S_{4}$ ). By (29) and Lemma 8, the remaining term in $s_{n}(x)$ is $O\left(\log \left(c^{2}-x^{2}\right)\right)$ almost everywhere, and so the corresponding term in $\sigma_{n}(x)$ is essentially bounded on $S_{7}$ which we now define as $S_{5}$ omitting a certain null set of values of $x$. This proves Lemma 9 . 
Theorem 3. The conclusion of Theorem 2 holds if the hypothesis that $f$ is essentially bounded is weakened to $f(x) \log \left(c^{2}-x^{2}\right) \in L(-c, c)$ and the condition (25) is strengthened to (29)

Proof. This is a modified version of the proof of Theorem 1. As in that proof, $s_{n}(t) \rightarrow g(t)$ almost everywhere. As in (30), this can be written

$$
\sigma_{n}(t) \log \left(c^{2}-t^{2}\right) \rightarrow g(t)
$$

almost everywhere. This gives that

$$
\int_{-c}^{c} f(t) s_{n}(t) d t=\int_{-c}^{c} f(t) \log \left(c^{2}-t^{2}\right) \sigma_{n}(t) d t \rightarrow \int_{-c}^{c} f(t) g(t) d t
$$

as $n \rightarrow \infty$, because $\sigma_{n}(t)$ converges boundedly by Lemma 9 and $f(t) \log \left(c^{2}-t^{2}\right)$ is integrable by hypothesis, so that their product converges dominatedly, a dominant function being a constant multiple of $f(t) \log \left(c^{2}-t^{2}\right)$.

Remark 2. The requirement on $f$ in Theorem 3 comes near to allowing $f$ to be any integrable function, which is the situation in the theorems for trigonometric Fourier series which were the inspiration for this paper.

Theorem 4. If $g$ is absolutely continuous on $[-c, c]$, Theorems 1 and 2 hold without the double integral requirement (25).

Proof. This consists in showing that (25) holds for all absolutely continuous g. If $t \neq x$,

$$
\left|\frac{g(t)-g(x)}{t-x}\right|=\left|\frac{1}{t-x} \int_{x}^{t} g^{\prime}(s) d s\right| \leq \frac{\operatorname{sgn}(t-x)}{|t-x|} \int_{x}^{t}\left|g^{\prime}(s)\right| d s=\frac{1}{t-x} \int_{x}^{t}\left|g^{\prime}(s)\right| d s
$$

so the double integral is at most

$$
\begin{gathered}
\int_{-c}^{c} d x \int_{-c}^{c} \frac{d t}{t-x} \int_{x}^{t}\left|g^{\prime}(s)\right| d s \\
=\int_{-c}^{c} d x \int_{-c}^{x} \frac{d t}{x-t} \int_{t}^{x}\left|g^{\prime}(s)\right| d s+\int_{-c}^{c} d x \int_{x}^{c} \frac{d t}{t-x} \int_{x}^{t}\left|g^{\prime}(s)\right| d s \\
=\int_{-c}^{c} d x \int_{-c}^{x}\left|g^{\prime}(s)\right| d s \int_{-c}^{s} \frac{d t}{x-t}+\int_{-c}^{c} d x \int_{x}^{c}\left|g^{\prime}(s)\right| d s \int_{s}^{c} \frac{d t}{t-x} \\
=\int_{-c}^{c} d x \int_{-c}^{x}\left|g^{\prime}(s)\right| \log \frac{x+c}{x-s} d s+\int_{-c}^{c} d x \int_{x}^{c}\left|g^{\prime}(s)\right| \log \frac{c-x}{s-x} d s
\end{gathered}
$$




$$
=\int_{-c}^{c}\left|g^{\prime}(s)\right| d s \int_{s}^{c} \log \frac{x+c}{x-s} d x+\int_{-c}^{c}\left|g^{\prime}(s)\right| d s \int_{-c}^{s} \log \frac{c-x}{s-x} d x .
$$

To show that the last two inner integrals are bounded functions of $s$, we have

$$
\int_{s}^{c} \log \frac{x+c}{x-s} d x=-2 c\left(\frac{c+s}{2 c} \log \frac{c+s}{2 c}+\frac{c-s}{2 c} \log \frac{c-s}{2 c}\right)=\int_{-c}^{s} \log \frac{c-x}{s-x} d x .
$$

Now for $-c \leq s \leq c, 0 \leq(c \pm s) /(2 c) \leq 1$; consequently, with upper signs corresponding,

$$
0 \geq \frac{c \pm s}{2 c} \log \frac{c \pm s}{2 c} \geq-e^{-1}
$$

since $0 \geq u \log u \geq-e^{-1}$ for $0 \leq u \leq 1$. Thus the two inner integrals each lie between 0 and $4 c / e$.

Finally this gives that the double integral in (25) is at most

$$
\frac{8 c}{e} \int_{-c}^{c}\left|g^{\prime}(s)\right| d s
$$

which is finite since $g^{\prime} \in L(-c, c)$.

\section{References}

[1] A. Erdélyi, W. Magnus, F. Oberhettinger, and F. G. Tricomi, Higher transcendental functions, vol. 1, Bateman Manuscript Project, Mc Graw-Hill, 1953, ch. III.

[2] E. R. Love and M. N. Hunter, Expansions in series of Legendre functions, Proc. London Math. Soc. (3) 64 (1992), 579-601.

[3] E. C. Titchmarsh, Theory of Functions, 2nd ed., Oxford University Press, 1939, ch. XIII. 